\title{
Mid-Infrared Silicate Variation in Long-Period, Oxygen-Rich Variable Stars
}

\author{
M. J. CREECH-EAKMAN and R. E. STENCEL \\ University of Denver, Denver CO, U.S.A.
}

We present preliminary results from our on-going monitoring campaign of a selected group of more than 30 Long-Period Variable (LPV) stars at 10, 11 and $18 \mu \mathrm{m}$. Our stars were chosen from a list by Little-Marenin \& Little (1990, $A J, 99,1173$ ) based upon a classification scheme of silicate features of oxygenrich LPV stars. We are monitoring these LPVs for changes in their silicate features at 10 and $18 \mu \mathrm{m}$ with respect to IR continuum and optical phase. We are attempting to ascertain the relationship of dust formation to optical period, and any shell-shock interactions from the acoustic shocks originating in the photosphere and later impinging on these dust-forming areas. The ultimate goal is to determine what conditions lead to dust formation and destruction in these environments, and whether or not an evolutionary sequence can be inferred for AGB objects based on their spectra and dust formation. The instrument being used for data acquisition is Denver University's (DU) TNTCAM (Ten aNd Twenty micron CAMera), a liquid-helium-cooled, mid-IR camera using a Rockwell $128 \times 128 \mathrm{Si}$ :As hybrid array and housing 7 filters on an externally driven filter wheel (Klebe et al. 1996, in Polarimetry of the Interstellar Medium). A portion of this list of stars was chosen for our initial campaign at the Wyoming Infrared Observatory (WIRO) in 1993, including the brightest of our objects with a range of periods and LML types (CreechEakman et al. 1997, ApJ, 477, 825). The rest of our list consists of a sample of LML types with periods of 300-400 days and fluxes of $25 \pm 5 \mathrm{Jy}$ at $8 \mu \mathrm{m}$ chosen for one of our ISO proposals. Ancillary mid-IR spectra exist from LRS on IRAS in 1983, our CAESR data from 1993, and CGS-3 data from UKIRT service time in 1995. We hope to obtain photometric data with TNTCAM and spectral data using ISO's sws, and TGIRS, DU's new Two Grating mid-IR Spectrometer.

We would like to acknowledge partial support under NASA grant NGT51290 . 